\title{
Commentary
}

\section{Current consensus on the management of fetal growth restriction}

\author{
M Patabendige ${ }^{a}$, A Jayawardane ${ }^{b}$
}

\begin{abstract}
Fetal growth restriction (FGR) refers to a fetus that has failed to reach its biological growth potential. Clinically, FGR is described as early or late-onset. Detecting signs of growth deterioration and determining the appropriate timing of fetal rescue delivery is challenging. In early-onset FGR, problems are related to prematurity whereas in late-onset FGR difficulty in diagnosis and resulting fetal mortality are the key problems for the clinician. This review summarizes pathophysiology, recent consensus on the classification of clinical phenotypes, screening, diagnosis, assessment of deterioration and surveillance, monitoring intervals, the timing of birth, neurodevelopment outcomes, term fetal growth restriction and place of cerebroplacental ratio in fetal growth restriction.
\end{abstract}

Key words: fetal growth restriction, management, biophysical profile, fetal doppler

Sri Lanka Journal of Obstetrics and Gynaecology 2020; 42: 53-60

DOI: http://doi.org/10.4038/sljog.v42i2.7942

\section{Introduction}

\section{Optimal growth and suboptimal growth}

Optimal fetal growth, i.e. a growth that is not too small and not too big, is classified as an estimated fetal weight (EFW) between the 10th and $90^{\text {th }}$ centiles based on population-specific birth weight centiles corrected for gestational age at delivery, parity, and fetal sex for ease of detection and management. With this definition, growth restriction is an EFW below the $10^{\text {th }}$ centile, better described as small for gestational age (SGA). Fetal growth restriction (FGR) has also been described as the process where a fetus that has a certain growth potential based on genetic criteria does not attain that growth because of environmental influence ['pathological smallness'] $]^{1}$. It is distinct from the term SGA

a Senior Registrar in Obstetrics and Gynaecology, Castle Street Hospital for Women, Colombo, Sri Lanka.

b Senior Lecturer in Obstetrics and Gynecology, Faculty of Medicine, University of Colombo, Sri Lanka.

Correspondence: MP, e-mail: mpatabendige@gmail.com

https://orcid.org/0000-0002-4092-7092

Received $30^{\text {th }}$ January 2020

Accepted 20 $0^{\text {th }}$ June 2020

This is an open-access article distributed under the terms of the Creative Commons Attribution 4.0 International License, which permits unrestricted use, distribution and reproduction in any medium provided the original author and source are credited. 
which describes both 'pathological and constitutional smallness"1. Since fetal weight depends on its gestational age, fetal dating scan has to be correct to prevent discrepancies from inaccurate dating.

SGA fetus carries an increased risk for stillbirth, and antenatal detection and recognition is inaccurate and suboptimal in most ${ }^{2}$. Antenatal recognition of FGR can halve stillbirth risk through earlier delivery of affected fetuses [fetal rescue delivery] ${ }^{2}$.

This review addresses pathophysiology, clinical phenotypes, surveillance and current consensus and new insights into the management of FGR.

\section{Pathophysiology}

\section{Early-onset FGR}

The classical concept is poor placentation leading to insufficient delivery of nutrients and oxygen to the fetus ${ }^{1}$. Uterine spiral arteries are physiologically remodelled into dilated inelastic tubes escaping maternal vasomotor control between 8-18 weeks during placenta formation, which results in a low-resistance uteroplacental circulation.

The pathophysiological pathways for defective placentation include immunological factors, endogenous vascular factors, and thrombogenic factors amongst others ${ }^{1}$. Poor placentation is associated with early-onset phenotype of both preeclampsia and FGR. Most of the women presenting with maternal hypertensive disorders before 34 weeks gestation will also have concurrent FGR. Sharing of a common placental pathology in both conditions may explain this phenomenon and thus confirming the concept of poor placentation ${ }^{1}$.

\section{Late-onset FGR}

In growth restriction presenting at later gestations, the previous association becomes less obvious. At or near term, most neonates from mothers with preeclampsia are of normal birth weight. Thus, both late FGR and late preeclampsia may be due to a different pathology ${ }^{1}$.
Hypotheses proposed include;

1. Secondary placental dysfunction: 'Crowded' large placenta compromising intervillous perfusion.

2. Maternal susceptibility hypothesis: maternal constitutional susceptibility, cardiovascular dysfunction or immunological response.

Because the term fetuses have less placental reserve capacity and require comparatively more oxygen and nutrients, the interval between the onset of the disease and the subsequent adverse outcome is shorter and less predictable ${ }^{1}$.

Late-onset FGR is a challenge to predict and harder to distinguish from pregnancies with normal growth. The defective placental maturational process leading to placental hypo-perfusion and late-onset FGR is difficult to diagnose because the size of the fetus and accompanying parameters may not be abnormal and further complicated by combinations and intermediate types ${ }^{1}$.

\section{Clinical phenotypes}

FGR evolves from a preclinical phase to a clinically obvious phase of growth delay and may eventually lead to fetal hypoxia ${ }^{3,4}$. Reduced growth due to decreased transplacental nutrient supply, gas transfer, and waste removal affect reserves, reflected in reduced fetal liver size and consequently abdominal circumference (AC) first, and subsequent head growth and finally affecting the entire body ${ }^{3,4}$. Abnormal placental perfusion in the maternal compartment results in increased blood flow resistance in the uterine artery, which is reflected in the Doppler flow-velocity waveform ${ }^{3,4}$. Abnormal perfusion of the fetal villous vascular tree is associated with decreased umbilical artery end-diastolic velocity (EDV) proportional to the degree of flow impairment ${ }^{3,4}$. Abnormal oxygen diffusion across the villous membrane leading to lower fetal arterial oxygen saturation $\left(\mathrm{PaO}_{2}\right)$ is associated with an attempted increase of blood supply through a decrease in middle cerebral artery (MCA) blood flow resistance $^{3,4}$.

Management challenges in early FGR and coexisting maternal hypertensive disease include iatrogenic prematurity whereas, in late FGR, difficulties in diagnosis and surveillance leading to unanticipated stillbirth are the primary issues. 


\section{Screening}

This is based on clinical findings correlated with ultrasound fetal biometry. Traditional clinical assessment of risk is based on medical and obstetrics history, abdominal palpation for fetal size and symphysis-fundal height (SFH) marked serially over a centile chart. Risk assessment can be done through detailed history according to a recommended standard such as RCOG algorithm or Growth Assessment Protocol algorithm ${ }^{2,5}$. Leopold's Maneuvers are used to estimate the fetal size. However, its ability to predict fetal weight is low, only $30 \%$ SGA fetuses will be detected ${ }^{6}$.

SFH is the most commonly used screening tool for SGA. SFH should be plotted against the gestational age starting from 24 weeks, then 28 weeks, then two weekly up to 36 weeks and weekly thereafter. SFH has a low sensitivity of $27-76 \%$ in predicting SGA, which means it potentially fails to identify over $70 \%$ of pregnancies affected by SGA ${ }^{7}$. This is important to consider in counselling of pregnant women ${ }^{7}$. In clinical practice, the SFH measurement should not be carried out in isolation and the combination of other clinical findings, medical conditions, and previous obstetric history, together will contribute to estimating the likelihood of being at risk for SGA. A higher specificity $(>80 \%)$ of $\mathrm{SFH}$ indicates that few pregnancies which are not characterized by SGA, are referred for ultrasound evaluation? ${ }^{7}$.

Serial SFH helps to predict the need for ultrasound parameters by lagging of fundus in a plotted chart with gestation specific centile curves. The sensitivity of SFH can be improved to $48 \%$ by using the customized charts [adjusted for physiological variables such as height, weight, parity, and ethnic origin] for the particular population, which reduces referrals for ultrasound assessment ${ }^{2}$. Customized charts also improve EFW assessment and reduce the false-positive diagnosis of SGA $^{2}$.

World Health Organization (WHO) charts, GROW (Gestation Related Optimal Weight) charts, INTERGROWTH-21 ${ }^{\text {st }}$ project is all different project towards standardizing of customized charts. WHO and GROW charts are widely accepted in clinical practice². GROW charts are useful when looking at subgroups within the population, e.g. maternal size. There is a better correlation between SGA defined by customized centiles and perinatal mortality than any population- based standard. GROW charts also improve the definition of SGA in multi-ethnic populations by reclassification of fetuses as normal growth according to customization ${ }^{8}$. These reclassified fetuses had the same perinatal mortality risk as to the general, nonSGA population further emphasizing the value of customized charts ${ }^{8}$.

The INTERGROWTH-21 $1^{\text {st }}$ project has been introduced as a one-size-fits-all standard for birth weight ${ }^{9}$. The INTERGROWTH-2 $1^{\text {st }}$ project was a large-scale, multicountry study that measured fetal growth of babies in utero and at birth, in mothers in whom environmental, social, medical and pregnancy conditions were optimal ${ }^{10}$. It followed low risk, well-nourished mothers, in eight countries and was therefore meant to provide an optimal standard for all pregnancies ${ }^{9,10}$. The INTERGROWTH-21 $1^{\text {st }}$ findings challenge the acceptance of customized fetal growth charts in the UK and elsewhere ${ }^{10}$. Adjusting growth expectations for ethnicity has no biological basis and risks normalizing suboptimal growth in disadvantaged groups ${ }^{10}$. However, this concept has been challenged on theoretical grounds ${ }^{1,11}$ and when applied to detailed multi-ethnic maternity data sets, it performed poorly compared to a customized standard ${ }^{2}, 12$.

\section{Diagnosis}

Serial ultrasound biometry and Doppler to confirm compromised circulation is the gold standard to diagnose FGR. Once diagnosed, monitoring is from umbilical Doppler flow (UAD), ductus venosus (DV) and middle cerebral artery (MCA) blood flow patterns. The combination of fetal biometry and UAD is the best available tool for the identification of an FGR fetus ${ }^{5}$. Uterine artery Doppler (UtAD) screening can be performed in 'high risk' cases [Thrombophilia, Previous FGR/Pre-eclampsia]. UtAD offers early evidence to prescribe low-dose aspirin in the first trimester and $85 \%$ sensitivity to predict SGA in the second trimester (22-23 weeks) in high-risk women. However, in 'low risk' mothers and after 24 weeks, it has limited predictive accuracy. Diagnosis can be based on fetal biometry alone or by considering UAD or MCA Doppler indices. Abdominal circumference (AC) less than $10^{\text {th }}$ centile has the highest sensitivity for the diagnosis of SGA, whereas EFW less than the $10^{\text {th }}$ centile is more specific ${ }^{13}$. Therefore serial EFW measurements are better than serial AC in detecting 
additional FGR, ${ }^{13}$. However, this has to be balanced against the potential +/- $10 \%$ margin of error in estimating EFW at term using ultrasound ${ }^{2}$.

The diagnostic cut-off for SGA is generally accepted as below $10^{\text {th }}$ centile. One disadvantage of this cut-off is the inclusion of a variable number of constitutionally small ("normal") fetuses that do not require surveillance ${ }^{2}$. These otherwise normal constitutionally small babies have comparable perinatal mortality to that of the normal population ${ }^{2}$. EFW less than $3^{\text {rd }}$ centile or a decreased AC is more likely to identify "true FGR". The disadvantage of taking $3^{\text {rd }}$ centile for cut-off is less severe form of FGR at risk for deterioration will be missed with the resulting risk of increased stillbirth. A combined consensus has been agreed on for diagnosis $^{14}$.
Although placental pathology does not support such clear-cut division, expert opinion considers FGR presenting before 32 weeks as “early-onset” and after 32 weeks as "late-onset" (Table 1). An estimated fetal weight less than the tenth percentile in association with an elevated umbilical artery Doppler index, a decreased middle cerebral artery Doppler index or a decreased cerebroplacental ratio (CPR) should be considered as evidence of FGR ${ }^{3}$. Early-onset and late-onset FGR represent two distinct clinical phenotypes of placental dysfunction. According to the Internal Society for Ultrasound in Obstetrics and Gynaecology (ISUOG) definition of FGR, “AC/EFW crossing centiles” can be defined as crossing $>2$ quartiles or $50^{\text {th }}$ centile as long as it occurs in a SGA fetus $\left(\mathrm{AC} / \mathrm{EFW}<10^{\text {th }}\right.$ centile) and/or in combination with a low CPR or elevated UAD Pulsatility Index ${ }^{14}$.

Table 1. Consensus-based definitions for early and late fetal growth restriction

\begin{tabular}{|c|c|}
\hline $\begin{array}{l}\text { Early-onset growth restriction } \\
\text { (in the absence of fetal anomalies) }\end{array}$ & $\begin{array}{l}\text { AC or EFW }<3^{\text {rd }} \text { centile or absent EDF in the } \\
\text { umbilical artery } \\
\text { or } \\
\text { 1) AC/EFW }<10^{\text {th }} \text { centile combined with } \\
\text { 2) Uterine artery PI }>95^{\text {th }} \text { centile and/or } \\
\text { 3) Umbilical artery PI }>95^{\text {th }} \text { centile }\end{array}$ \\
\hline $\begin{array}{l}\text { Late-onset growth restriction } \\
\text { (in the absence of fetal anomalies) }\end{array}$ & $\begin{array}{l}\text { AC or EFW }<3^{\text {rd }} \text { centile } \\
\text { or } \\
\text { At least two of three of the following: } \\
\text { 1) AC/EFW }<10^{\text {th }} \text { centile } \\
\text { 2) Crossing centiles* of more than two quartiles } \\
\text { on growth centiles } \\
\text { 3) } \mathrm{CPR}<5^{\text {th }} \text { centile }\end{array}$ \\
\hline
\end{tabular}

*AC/EFW crossing centiles can be defined as crossing $>2$ quartiles or $50^{\text {th }}$ centile as long as it occurs in a SGA fetus. $A C$, abdominal circumference; CPR, cerebroplacental ratio; EDF, end-diastolic flow; EFW, estimated fetal weight; $P I$, pulsatility index. Source: Adapted from Gordijn et $\mathrm{al}^{14}$. 


\section{Assessment of the degree of fetal deterioration}

Gestational age is a critical determinant in decision making. Fetal surveillance tests are applied in pregnancies with suspected FGR to estimate the risk for hypoxia, prelabor acidosis, and antepartum stillbirth, as well as the clinical deterioration. An accurate estimation of hypoxia/ acidosis is important to predict fetal compromise, risk of stillbirth and therefore the timing of delivery ${ }^{3,4}$. Doppler indices and biophysical profile (BPP) provide estimates for pre-labor acidosis.

Integrated multimodal fetal testing is probably the way forward compared to the elusive single best test of fetal well-being. Tests include kick count charts, clinical examination, Doppler parameters, and BPP. Abnormal umbilical flow patterns indicate an increased risk of hypoxia and acidosis. Fetal acidosis is directly proportional to the reduction in umbilical artery EDV with acidosis highest in reversed flow in end-diastole. However umbilical artery Doppler alone as a single test is not justified due to the wide range of $\mathrm{pH}$ distribution and additional Doppler indices are indicated depending on the gestation.

DV Doppler and presence of 'a' wave for forwarding blood floware indicated up to 32 weeks for fetuses with absent or reversed umbilical artery Doppler indices. MCA Doppler has limited accuracy to predict acidosis before 32 weeks and adverse outcomes and should not be used to time delivery in early FGR. Beyond 3234 weeks, the umbilical artery waveform may be normal with developing acidosis. Therefore, better predictors for fetal hypoxia and deterioration is MCA PI and cerebroplacental ratio (CPR) beyond 32 weeks, which is considered as abnormal if less than $5^{\text {th }}$ centile.

BPP shows a reliable and reproducible relationship with the fetal $\mathrm{pH}$, irrespective of gestational age $\mathrm{e}^{3,4}$. Each component in BPP is independently altered by hypoxia and it takes minimum 30 minutes of work to assess one fetus ${ }^{3,4}$. It can be combined with computerized CTG (cCTG). Abnormal CTG is associated with a wide range of $\mathrm{pH}$ values, unlike cCTG. However, cCTG as a stand-alone test in FGR offers limited accuracy. BPP parameters (especially loss of tone) have a narrower $\mathrm{pH}$ distribution in acidosis when compared to Doppler ${ }^{3,4}$. BPP alone has limited utility in the prediction of longitudinal deterioration, which is better assessed with Doppler $^{15,16}$.

\section{Selection of the monitoring intervals}

Fetal surveillance is indicated to prevent stillbirth and irreversible fetal deterioration by early detection of prelabor acidosis and carry out a fetal rescue delivery balancing against neonatal morbidity and mortality of preterm birth. The consensus is based on expert opinion due to a lack of high-quality evidence to guide practice $^{3}$. Once FGR is diagnosed, longitudinal surveillance starting at 24 to 26 weeks with integrated multimodal fetal testing, including multi vessel Doppler assessment, fetal heart rate analysis, and BPP is considered best practice ${ }^{3,4}$. It is expected that the combination of these tests improves the prediction of acidosis and stillbirth compared with single tests as outlined above.

In early FGR, fetal needs are less and allow a relatively chronic course leading to a small baby. Fetal deterioration typically evolves from abnormal umbilical artery Doppler studies to brain-sparing, then abnormal ductus venous Doppler, abnormal cCTG, and finally, abnormal BPP. The rate of progression is determined by the interval between diagnosis to loss of umbilical artery EDV which typically takes four to six weeks. Once forward velocities in the DV Doppler become absent or reversed, fetal survival of longer than one week is unlikely ${ }^{17}$.

In late FGR, risk of terminal deterioration and unanticipated stillbirth is high, warranting closer surveillance. There are no evident Doppler changes in the precordial veins [pre-load to the fetal heart] and brain sparing may be the only observed Doppler sign of hypoxia. The new onset of Doppler abnormalities signals to expedite the birth ${ }^{3,4}$. Closer surveillance is required after 34 weeks because more fetal needs, less placental reserve and less time to adapt.

\section{Planning birth}

Gestational age should be the intervention threshold. Neonatal survival, more importantly neurologically intact survival, improves with gestational age. Planning birth is a decision taken after balancing the risks and benefits of birth compared to the continuation of pregnancy.

1. 24 to 26 weeks: Even with the best available care, the survival rate of growth-restricted neonates average less than $50 \%{ }^{18}$. In the 
surviving babies, risks for major neonatal morbidity is high as $80 \%$. The survival rates exceed $50 \%$ once the EFW exceeds $500 \mathrm{~g}$ and/or when 26 weeks have reached. Delivery at this age is more commonly due to maternal life-threatening conditions such as severe preeclampsia than FGR.

2. 26 to 28 weeks: Neonatal survival exceeds $50 \%$. However, intact survival (survival without prematurity-related morbidity) at 26 to 27 weeks remains around $30 \%$. Ductus venous Doppler abnormalities are not considered as an indication to birth until 28 weeks $^{3}$ while abnormal BPP $(<6 / 10)$ is an indication for delivery. Each day in utero increases neonatal survival by a median of $2 \%{ }^{3}$.

3. 28 to 32 weeks: Neonatal survival exceeds $70 \%$ at 28 weeks and increases to more than $90 \%$ at 32 weeks. Fetal deterioration of venous Doppler parameters may be tolerated as long as DV flow is forward. Reversal of the DV a-wave before delivery reduces the chance of neonatal survival. Persistence of this abnormality beyond one week carries a significant risk for stillbirth. For this reason, the presence of a DV reversed a-wave is generally considered an indication of intervention from 28 weeks. However, birth before 30 weeks gestation still carries a significantly higher risk for adverse neurodevelopment at age two because of neonatal complications and their impact on motor development. Each day in utero increases neonatal survival by a median of $1 \%$. These conclusions were based on TRUFFLE and GRIT trials and their follow up data ${ }^{19,20,21,22}$. Although there is no difference in neonatal mortality between immediate and deferred deliveries, due to a significant difference in morbidity in an immediate delivery group of early FGR, delaying at least up to 32 weeks is generally advocated based on these TRUFFLE and GRIT data ${ }^{19,20,21,22}$.

4. 32 to 34 weeks: Cerebral circulation gains an additional structural layer reducing cerebral haemorrhage risk during this period. This reduction has a measurable impact on motor development at age three. Administration of antenatal steroids has an added benefit in reducing respiratory morbidity and cerebral haemorrhage, and babies who have received steroids have improved survival. Evidence suggests that neurodevelopment is also improved by the administration of steroids ${ }^{23}$. Umbilical artery absent/reversed EDV is generally considered an indication for birth from 32 weeks onwards.

5. 34 to 38 weeks: Gain in survival as well as neonatal morbidity is minimal. However, up to 38 weeks gestation, the rate of neonatal admissions to the NICU is still significantly greater for FGR infants. Umbilical artery absent/reversed EDV is an indication of delivery from 34 weeks onwards. MCA Doppler is considered the best predictor of fetal adaptation to hypoxia, and some guidelines recommend the use of this parameter to time delivery in fetuses with normal umbilical artery Doppler ${ }^{5}$.

6. After 38 weeks: Neonatal adverse events in SGA infants are negligible and, accordingly, on-going pregnancy must be weighed carefully against the risks of unanticipated stillbirth if the patient remains undelivered. DIGITAT study has shown among women with suspected FGR at 36-41 weeks, a policy of labour induction affects neither the rate of adverse neonatal outcomes nor the rates of instrumental vaginal delivery or caesarean delivery, indicating that both approaches are acceptable ${ }^{24}$. PROBAAT study group has shown that the use of Foley catheters for labour induction offers a better outcome compared to prostaglandins for FGR fetuses ${ }^{25}$. The consensus from the DIGITAT trial is that the optimum time for induction in SGA with normal Doppler study is around 38 weeks because it is associated with the lowest neonatal morbidity and seems to minimize the risk of stillbirth ${ }^{26}$.

Between 24 and 34 weeks, a single course of steroids should be given over 48 hours if birth is being considered in the next seven days. Magnesium sulphate for fetal neuroprotection needs to be considered before 
34 weeks which halves the risk of cerebral palsy. Birth should be planned at a centre with neonatal intensive care facilities and mode of delivery depends on the severity of fetal compromise, along with other maternal and obstetric factors. In FGR cases with abnormal $\mathrm{UAD}$, induction of labour can be offered, but the rate of emergency caesarean birth is high. Because of the increased risk of intrapartum asphyxia in FGR, continuous FHR monitoring is recommended from the onset of uterine contractions.

\section{Neurodevelopmental outcomes in FGR}

In early FGR, particularly before 28 weeks, gestational age at birth and birth weight are the most important predictors for poor neurodevelopment, advanced cardiovascular deterioration, and ICH leading to psychomotor retardation. Whereas in late FGR, gestational age at birth is a smaller contributor and other main predictors have yet to be defined. It mainly affects behavioral rather than motor development.

\section{Cerebroplacental ratio and 'term FGR'}

Many groups have suggested the CPR as a functional marker of failure to reach growth potential at or near term $^{26}$. Low CPR, regardless of the fetal size, is independently associated with the need for operative birth for presumed fetal compromise and neonatal unit admission at term ${ }^{26}$. Rising evidence proposes that CPR as a marker of impaired fetal growth velocity and adverse pregnancy outcome, even in fetuses whose size is considered "appropriate for gestational age" using conventional fetal biometry ${ }^{26,27}$. Therefore it is wise to apply CPR at 40 weeks assessment of lowrisk pregnancies with fetal biometry to assess the suitability to wait a few more days without intervention.

\section{Conclusion}

Early FGR is associated with significant uteroplacental blood flow abnormalities and abnormal cardiovascular findings with evolving deterioration. In contrast, late FGR is not dominantly associated with placental vascular blood flow abnormalities and shows minimal signs of deterioration making it difficult to predict. EFW below the $10^{\text {th }}$ centile with co-existing UAD and/ or MCA Doppler flow and/or CPR abnormalities can identify a small fetus with pathological growth restriction requiring antenatal surveillance and planning delivery. Gestational age is the primary determinant as the threshold for intervention in planning delivery. Decision of timing of delivery is a balanced decision after considering fetal risks of continuing pregnancy and neonatal risks after delivery.

\section{Author Declarations}

Competing interests: The authors declare that they have no competing interests.

Funding: No funding sources received.

Authors' Contributions: First author, MP did the conception, design, data collection, analysis and writing/editing of the paper and he is the principal author for this work. Author AJ participated in writing and editing of the paper. Both authors approved the final manuscript to be published.

\section{References}

1. Lees C, Visser GHA, Hecher K. Placental-Fetal Growth Restriction. Cambridge University Press 2018: Cambridge CB2 8BS, United Kingdom 9781-107-10139-5.

2. Williams M, Turner S, Butler E, Gardosi J. Fetal growth surveillance - Current guidelines, practices and challenges. Ultrasound 2018; 26(2): 69-79.

3. Seravalli V, Baschat AA. A uniform management approach to optimize outcome in fetal growth restriction. Obstet Gynecol Clin North Am. 2015; 42(2): 275-88.

4. Baschat AA. Planning management and delivery of the growth-restricted fetus. Best Pract Res Clin Obstet Gynaecol. 2018; 49: 53-65.

5. Royal College of Obstetricians and Gynaecologists. The Investigation and Management of the Smallfor-Gestational-Age Fetus. Green-top Guideline No. 31. 2014.

6. Tejani N, Mann LI. Diagnosis and management of the small-for-gestational-age fetus. Clin Obstet Gynecol 1977; 20: 943-55.

7. Pay AS, Wiik J, Backe B, Jacobsson B, Strandell A, Klovning A. Symphysis-fundus height measurement to predict small-for-gestational-age status at birth: a systematic review. BMC Pregnancy Childbirth. 2015; 15: 22. 
8. Giddings S, Clifford S, Madurasinghe V, et al. PFM.69 Customised vs uncustomised ultrasound charts in the assessment of perinatal mortality risk in the South Asian maternity population. Arch Dis Child 2014; 99 (Suppl 1): A104-A104.

9. Villar J, Ismail LC, Victora CG, et al. International standards for newborn weight, length, and head circumference by gestational age and sex: the newborn cross-sectional study of the INTERGROWTH-21st project. Lancet 2014; 384: 857-868.

10. Hirst JE, Papageorghiou AT. INTERGROWTH21st: a new paradigm for fetal growth in the 21st century. The Obstetrician \& Gynaecologist 2016; 18: $137-41$.

11. Hanson M, Kiserud T, Visser GHA, et al. Optimal fetal growth: a misconception? Am J Obstet Gynecol 2015; 213: 332.e1-4.

12. Anderson NH, Sadler LC, McKinlay CJD, et al. INTERGROWTH-21st vs customized birthweight standards for identification of perinatal mortality and morbidity. Am J Obstet Gynecol 2016; 214: 509. e1-7.

13. Baschat AA, Weiner CP. Umbilical artery doppler screening for detection of the small fetus in need of antepartum surveillance. Am J Obstet Gynecol 2000; 182(1 Pt 1): 154-8.

14. Gordijn SJ, Beune IM, Thilaganathan B et al. Consensus definition of fetal growth restriction: a Delphi procedure. Ultrasound Obstet Gynecol 2016; 48: 333-339.

15. Baschat AA. Integrated fetal testing in growth restriction: combining multivessel Doppler and biophysical parameters. Ultrasound Obstet Gynecol 2003; 21(1): 1-8.

16. Baschat AA, Gembruch U, Harman CR. The sequence of changes in Doppler and biophysical parameters as severe fetal growth restriction worsens. Ultrasound Obstet Gynecol 2001; 18(6): 571-7.

17. Turan OM, Turan S, Berg C, et al. Duration of persistent abnormal ductus venosus flow and its impact on perinatal outcome in fetal growth restriction. Ultrasound Obstet Gynecol 2011; 38(3): 295-302.

18. Baschat AA, Cosmi E, Bilardo CM, et al. Predictors of neonatal outcome in early onset placental dysfunction. Obstet Gynecol 2007; 109(2 Pt 1): 253-61.

19. Bilardo CM, Hecher K, Visser GHA, Papageorghiou AT, Marlow N, Thilaganathan B, et al. Severe fetal growth restriction at 26-32 weeks: key messages from the TRUFFLE study. Ultrasound Obstet Gynecol. 2017; 50(3): 285-90.

20. Frusca T, Todros T, Lees C, Bilardo CM; TRUFFLE Investigators. Outcome in early-onset fetal growth restriction is best combining computerized fetal heart rate analysis with ductus venosus Doppler: insights from the Trial of Umbilical and Fetal Flow in Europe. Am J Obstet Gynecol. 2018; 218(2S): S783-S789.

21. Walker D-M, Marlow N, Upstone L, et al. The Growth Restriction Intervention Trial: long-term outcomes in a randomized trial of timing of delivery in fetal growth restriction. Am J Obstet Gynecol 2011; 204: 34.e1-9

22. Thornton JG, Hornbuckle J, Vail A, Spiegelhalter DJ, Levene M; GRIT study group. Infant wellbeing at 2 years of age in the Growth Restriction Intervention Trial (GRIT): multicentred randomised controlled trial. Lancet. 2004; 364(9433): 513-20.

23. Sotiriadis A, Tsiami A, Papatheodorou S, et al. Neurodevelopmental outcome after a single course of antenatal steroids in preterm infants: a systematic review and meta-analysis. Obstet Gynecol. 2015; 125(6): 1385-96.

24. Boers KE, Vijgen SM, Bijlenga D, et al. Induction versus expectant monitoring for intrauterine growth restriction at term: randomised equivalence trial (DIGITAT). BMJ 2010; 341: c7087.

25. Jozwiak M, et al. Foley catheter versus vaginal prostaglandin E2 gel for induction of labour at term (PROBAAT trial): an open-label, randomised controlled trial. Lancet. 2011; 378: 2095-103.

26. Boers KE, van Wyk L, van der Post JA, et al. Neonatal morbidity after induction vs expectant monitoring in intrauterine growth restriction at term: a subanalysis of the DIGITAT RCT. Am J Obstet Gynecol 2012; 206(4): 344.e1-7.

27. Khalil A, Morales-Rosello J, Khan N, Nath M, Agarwal P, Bhide A, Papageorghiou A, Thilaganathan $\mathrm{B}$. Is cerebroplacental ratio a marker of impaired fetal growth velocity and adverse pregnancy outcome? Am J Obstet Gynecol 2017; 216(6): 606.e1-606.e10. 